\title{
Cause Traced to Environment
}

National Cancer Institute

\section{Source}

National Cancer Institute. Cause Traced to Environment. NCI Thesaurus. Code C139473.

Problems caused by exposure to environmental conditions outside the expected range. 